\title{
Resonance Y-shape Solitons and Mixed Solutions for a (2+1)-dimensional Generalized Caudrey-Dodd- Gibbon-Kotera-Sawada Equation in Fluid Mechanics
}

hongcai ma ( $\sim$ hongcaima@hotmail.com )

Donghua University https://orcid.org/0000-0001-8759-5032

Shupan Yue

Donghua University

Aiping Deng

Donghua University

\section{Research Article}

Keywords: (2+1)-dimensional gCDGKS equation, a new constrained condition, resonance Y-type soliton, hybrid solutions

Posted Date: December 20th, 2021

DOI: https://doi.org/10.21203/rs.3.rs-1177569/v1

License: (c) (i) This work is licensed under a Creative Commons Attribution 4.0 International License. Read Full License

Version of Record: A version of this preprint was published at Nonlinear Dynamics on January 19th, 2022. See the published version at https://doi.org/10.1007/s11071-022-07205-z. 


\title{
Resonance Y-shape solitons and mixed solutions for a $(2+1)$-dimensional generalized Caudrey-Dodd-Gibbon-Kotera-Sawada equation in fluid mechanics
}

\author{
Hongcai Ma ${ }^{1,2 *}$, Shupan Yue ${ }^{1 \dagger}$ and Aiping Deng ${ }^{1,2}$ \\ ${ }^{1}$ Department of Applied Mathematics, Donghua University, Shanghai 201620, China \\ ${ }^{2}$ Institute for Nonlinear Sciences, Donghua University, Shanghai 201620, China
}

\begin{abstract}
Under the well-known bilinear method of Hirota, the specific expression for N-soliton solutions of $(2+1)$-dimensional generalized Caudrey-Dodd-Gibbon-Kotera-Sawada(gCDGKS) equation in fluid mechanics is given. By defining a noval restrictive condition on $\mathrm{N}$-soliton solutions, resonant $\mathrm{Y}$-type and $\mathrm{X}$-type soliton solutions are generated. Under the previous new constraints, combined with the velocity resonance method and module resonant method, the mixed solutions of resonant Y-type solitons and line waves, breather solutions are found. Finally, with the support of long wave limit method, the interaction between resonant Ytype solitons and higher-order lumps is shown, and the motion trajectory equation before and after the interaction between lumps and resonant Y-type solitons is derived.
\end{abstract}

Keywords: (2+1)-dimensional gCDGKS equation; a new constrained condition; resonance Y-type soliton; hybrid solutions

\section{Introduction}

As a local nonlinear wave, solitons have many interesting properties [1]. By adding some constraints on the N-soliton solution obtained by Hirota bilinear method, one can obtain many types of bound molecules. Through the velocity resonance mechanism, the soliton molecular solution first observed in physical experiments is obtained [2-6]. By using the module resonant method, the breather solution which changes periodically with time can be gained $[7,8]$. Through long wave limit approach, one can gain lump solution $[9,10]$. By combining the above methods, one can also get a hybrid solution consisting of soliton molecules, breather solutions and lump solutions [11-16].

Resonant soliton is a special kind of soliton existing in integrable system, which has been widely studied. As we all know, If phase shift of the colliding solitons becomes infinite or converges to infinity, solitons will resonate [17]. The well-known soliton fusion and fission is a resonance phenomenon $[18,19]$. When it comes to the resonant Y-type soliton solutions, people

\footnotetext{
${ }^{*}$ Correspondence should be addressed to Hongcai Ma: Email addresses: hongcaima@hotmail.com

${ }^{\dagger}$ Email addresses: spyue0111@163.com
} 
usually directly restrict N-soliton solution derived from Hirota bilinear method. At first, most people set the form of the solution $[20,21]$ to

$$
u=\operatorname{aln}\left(1+\sum_{j=1}^{N} e^{k_{j} x+r_{j} y+w_{j} t+\phi_{j}}\right)_{x x},
$$

however, due to many formal constraints, the form of the solution is a little simple, so it is very laborious to obtain interactions between resonant Y-shaped solitons and other solutions. Later, Chen and others constructed another constraint, which obtained the mixed solutions of resonant Y-type soliton and 1-order lump [22]. The resonant multi soliton solution can be obtained by the linear superposition principle [23]. Recently, in order to obtain more interaction solutions between resonant Y-type solitons and other solutions, Li et al proposed a new constraint [24-27]. By this method, we can obtain the mixed solutions of resonant Y-shaped solitons and line waves, higher-order lumps, breather solutions and resonant Y-shaped solitons respectively.

A (2+1)-dimensional gCDGKS equation [28] is derived

$$
\begin{aligned}
& 36 u_{t}+\left(u_{4 x}+15 u u_{x x}+15 u^{3}\right)_{x}-\alpha v_{y y}-\beta\left(u_{x x y}+3 u u_{y}+3 u_{x} v_{y}\right)=0 \\
& u=v_{x}
\end{aligned}
$$

where $\alpha$ and $\beta$ are two arbitrary constants. For equation (1), Deng et al have obtained Nth-order Pfaffian and periodic wave solutions [29], Peng et al have also derived solitary and lump waves and their interaction phenomena [28].

In fluid mechanics, if $\alpha=5, \beta=5$, equation (1) will be reduced to $(2+1)$-dimensional CDGKS equation,

$$
\begin{aligned}
& 36 u_{t}+\left(u_{4 x}+15 u u_{x x}+15 u^{3}\right)_{x}-5 v_{y y}-5\left(u_{x x y}+3 u u_{y}+3 u_{x} v_{y}\right)=0 \\
& u=v_{x}
\end{aligned}
$$

which was first introduced in [30]. In addition, quasi-periodic solutions [31], diverse solitons, interaction solutions [32], rogue waves, generalized and classical lump solutions [33] of (2+1)dimensional CDGKS equation have been obtained.

If $\alpha=5, \beta=5$ and $u_{y}=0$, equation (1) will be reduced to $(1+1)$-dimensional CDGKS equation.

If $\alpha=5, t=36 T, \beta=5$ and $u_{y}=0$, equation (1) will be reduced to Sawada-Kotera equation for the long waves in shallow water under the gravity, which have gained many interesting results [34-36]. And also be used in lattice dynamics, quantum mechanics and nonlinear optics.

This article is divided into five sections to describe our content. In the second section, Nsoliton solution for equation (1) is given directly with Hirota bilinear method. The third section is divided into two parts. In the first part, based on $\mathrm{N}$-soliton solution and taking advantage of new constraints, we obtain the resonant Y-shaped solitons and some noval interaction solutions, especially the resonant X-type solution; In the second part, mixed solution of resonant Y-shaped soliton and line wave, breather wave are obtained under new constraints by velocity resonance and mode resonance methods. In section 4 , with the support of long wave limit approach, the interaction between resonant Y-type solitons and higher-order lumps is shown, and the motion trajectory equation before and after the interaction between lumps and resonant Y-type solitons is dissussed detailly. The fifth section is a short conclusion. 


\section{N-soliton solution}

Using logarithmic transformation

$$
u=2(\ln f)_{x x},
$$

the equation (1) can be transformed into the following bilinear equation:

$$
\left(36 D_{x} D_{t}+D_{x}^{6}-\alpha D_{y}^{2}-\beta D_{x}^{3} D_{y}\right)(f \cdot f)=0,
$$

where $D_{x}, D_{y}, D_{t}$ are defined as follows [37]:

$$
D_{x}^{m_{1}} D_{y}^{m_{2}} D_{t}^{m_{3}}(f \cdot f)=\left.\left(\partial x-\partial x^{\prime}\right)^{m_{1}}\left(\partial y-\partial y^{\prime}\right)^{m_{2}}\left(\partial t-\partial t^{\prime}\right)^{m_{3}}(f \cdot f)\right|_{x^{\prime}=x, y^{\prime}=y, t^{\prime}=t} .
$$

For the sake of obtaining the N-soliton solution, based on famous bilinear method, $f$ in $(2)$ is directly constructed as

$$
f=\sum_{\mu=0,1} \exp \left(\sum_{j=1}^{N} \mu_{j} \eta_{j}+\sum_{j<s}^{N} \mu_{j} \mu_{s} A_{j s}\right)
$$

with

$$
\begin{gathered}
\eta_{j}=k_{j} x+r_{j} y+w_{j} t+\phi_{j}, \\
w_{j}=\frac{-k_{j}^{6}+\beta k_{j}^{3} r_{j}+\alpha r_{j}^{2}}{36 k_{j}}
\end{gathered}
$$

and

$$
e^{A_{j s}}=-\frac{36\left(k_{j}-k_{s}\right)\left(w_{j}-w_{s}\right)+\left(k_{j}-k_{s}\right)^{6}-\alpha\left(r_{j}-r_{s}\right)^{2}-\beta\left(k_{j}-k_{s}\right)^{3}\left(r_{j}-r_{s}\right)}{36\left(k_{j}+k_{s}\right)\left(w_{j}+w_{s}\right)+\left(k_{j}+k_{s}\right)^{6}-\alpha\left(r_{j}+r_{s}\right)^{2}-\beta\left(k_{j}+k_{s}\right)^{3}\left(r_{j}+r_{s}\right)} .
$$

Here, $k_{j}, r_{j}$ and $\phi_{j}$ are arbitrary constants, $\sum_{\mu=0,1}$ is all possible combinations of $u_{i}=0,1(i=$ $1, \ldots, N)$.

\section{Resonant $\mathrm{Y}$-shape soliton and interactions}

\subsection{Resonance Y-shape soliton}

On the basis of $\exp (x+\ln (0))=0 \exp (x)=0$, we can eliminate some terms of equation (3). Therefore, in order to obtain mixed solutions of L-resonance Y-type soliton and P-resonance Y-type soliton, we need to make the following constraints on certain parameters for the above N-soliton solution:

$$
e^{A_{j s}}=0(N=L+P, 1 \leq j<s \leq L, L<j<s \leq N)
$$

where

$$
\begin{aligned}
r_{s}= & \frac{\beta k_{j}^{3}-3 \beta k_{j}^{2} k_{s}+2 \beta k_{j} k_{s}^{2}+2 \alpha r_{j}}{2 \alpha k_{j}} k_{s} \\
& \pm \frac{\sqrt{\left(k_{j}-k_{s}\right)^{2} k_{j}\left[\left(\beta^{2}-20 \alpha\right) k_{j}^{3}-4 k_{j} k_{s}\left(k_{j}-k_{s}\right)\left(\beta^{2}-5 \alpha\right)+12 \beta \alpha r_{j}\right]}}{2 \alpha k_{j}} k_{s} .
\end{aligned}
$$

Obviously, it can be seen from the expression of $r_{s}$ that there are positive and negative cases in equation (7), which explains that there will be two opposite cases for resonant Y-type soliton. 
When $N=2$, we take the parameters in (2) as $\alpha=1, \beta=5, k_{1}=\frac{1}{2}, k_{2}=\frac{1}{3}, r_{1}=1, r_{2}=$ $\frac{139}{216} \pm \frac{29 \sqrt{5}}{216}, \phi_{1}=0, \phi_{2}=0$ when $t=0$, and its density and three-dimensional plot are in figure a, b, d, e of Fig. 1.

When $N=3$, we take the parameters in (2) as $\alpha=-1, \beta=-2, k_{1}=\frac{1}{2}, k_{2}=\frac{1}{4}, k_{3}=$ $\frac{1}{5}, r_{1}=\frac{1}{2}, r_{2}=\frac{1}{4}-\frac{\sqrt{111}}{64}, r_{3}=\frac{1}{3}, \phi_{1}=0, \phi_{2}=0, \phi_{3}=0$ when $t=0$, and its density and three-dimensional plot are in figure c, f of Fig. 1.

As shown in Fig. $1(\mathrm{a}, \mathrm{d})$ and Fig. 1 (b,e), two 2-resonant Y-type soliton openings have different directions, and only $r_{2}$ of their parameters are different. In addition, Fig. 1 (c,f) gives a 3-resonant Y-type soliton.

If $N=4$, i.e. $L=2, P=2$, one can get the expression describing the interaction phenomenon of two 2-resonant Y-type soliton is

$$
u_{1}=2\left(\ln f_{1}\right)_{x x}
$$

with

$$
f_{1}=1+e^{\eta_{1}}+e^{\eta_{1}+\eta_{3}+A_{13}}+e^{\eta_{2}}+e^{\eta_{1}+\eta_{4}+A_{14}}+e^{\eta_{3}}+e^{\eta_{2}+\eta_{3}+A_{23}}+e^{\eta_{4}}+e^{\eta_{2}+\eta_{4}+A_{24}} .
$$

Here parameters $e^{\eta_{j}}$ and $e^{A_{j s}}$ are limited by (4),(5),(6), and (7).

Via selecting appropriate constants, there are three main situations for interaction between two 2-resonant Y-shape soliton. Fig. 2, Fig. 3 and Fig. 4 show detailly the interaction phenomena of resonance Y-shape solutions with two fission, two fusion, and one fission with one fusion. Because the interaction between line waves is elastic and resonant Y-type solitons are simplified by $\mathrm{N}$-soliton solutions, interaction between resonant $\mathrm{Y}$-shape soliton is also elastic.

If we take

$$
u_{2}=2\left(\ln f_{2}\right)_{x x},
$$

where

$$
f_{2}=1+e^{\eta_{1}}+e^{\eta_{2}}+e^{\eta_{3}}+e^{\eta_{2}+\eta_{3}+A_{23}},
$$

and $r_{2}, r_{3}$ and $e^{A_{23}}$ are determined respectively by conditions (7) and (5), one can get a resonant $\mathrm{X}$-type soliton, which is formed by two resonant Y-shape soliton with opposite opening directions. It is a new type of double open resonant Y-type soliton. As shown in Fig. 5, the double open resonant Y-type solitons are one in fission and one in fusion.

\subsection{Interaction between line wave molecule, breather and resonant Y-type soliton}

Based on the local velocity resonance method and constraints condition (7), a method to directly obtain the interaction between resonant Y-type solitons and line solitons is proposed. The following conditions are set on the $\mathrm{N}$-soliton solution (3):

$$
\begin{aligned}
& e^{A_{j s}}=0, \frac{k_{L+2 p-1}}{k_{L+2 p}}=\frac{r_{L+2 p-1}}{r_{L+2 p}}=\frac{w_{L+2 p-1}}{w_{L+2 p}} \neq \pm 1, \\
& (1 \leq j<s \leq L, 1 \leq p \leq P, N=L+2 P) .
\end{aligned}
$$

If $L=2, P=1$, we will gain a hybrid solution of a line soliton and 2-resonant Y-type soliton of equation (1), which is expressed as

$$
u_{3}=2\left(\ln f_{3}\right)_{x x}
$$




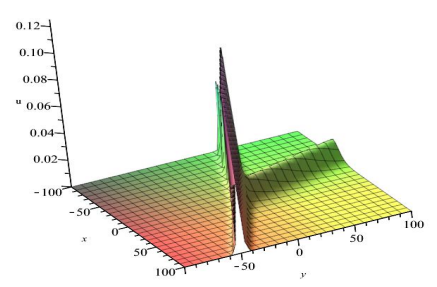

(a)

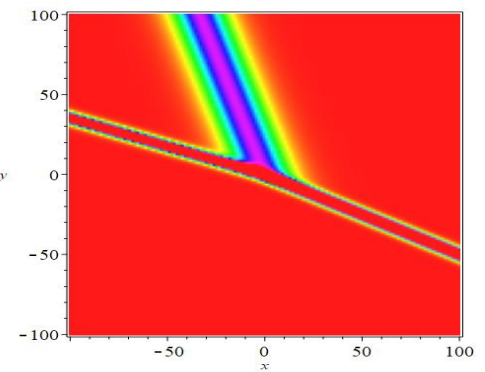

(d)

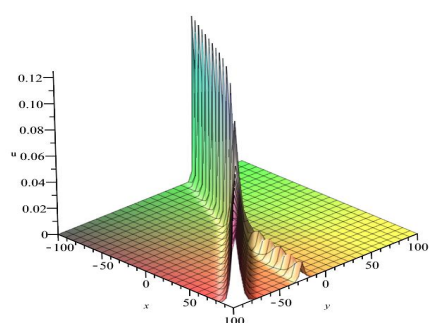

(b)

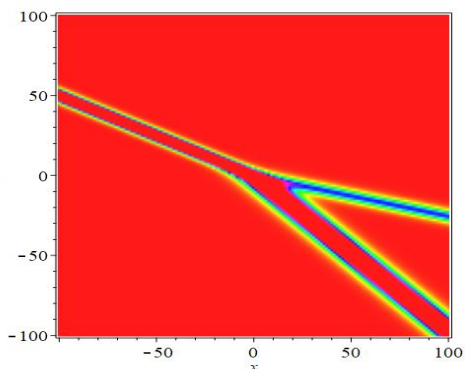

(e)

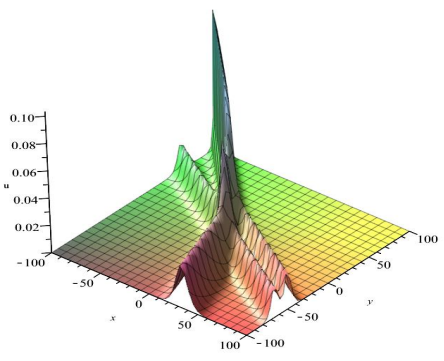

(c)

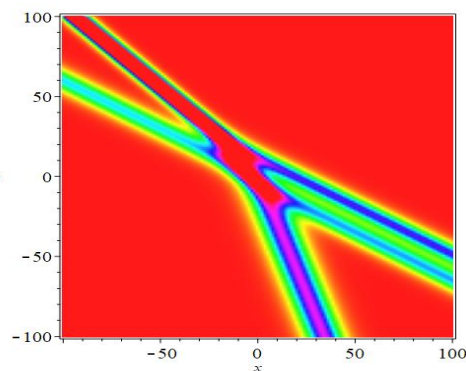

(f)

Figure 1: A 2-resonance Y-shape soliton of (2) with $\alpha=1, \beta=5, k_{1}=\frac{1}{2}, k_{2}=\frac{1}{3}, r_{1}=1, \phi_{1}=0, \phi_{2}=0$ when $t=0$, and:(a,d) $r_{2}=$ $\frac{139}{216}+\frac{29 \sqrt{5}}{216} ;(\mathrm{b}, \mathrm{e}) r_{2}=\frac{139}{216}-\frac{29 \sqrt{5}}{216}$. 3-resonance Y-shape soliton of (2) with $\alpha=-1, \beta=-2, k_{1}=\frac{1}{2}, k_{2}=\frac{1}{4}, k_{3}=\frac{1}{5}, r_{1}=\frac{1}{2}, r_{2}=$ $\frac{1}{4}-\frac{\sqrt{111}}{64}, r_{3}=\frac{1}{3}, \phi_{1}=0, \phi_{2}=0, \phi_{3}=0$ when $t=0$ in $(\mathrm{c}, \mathrm{f})$.

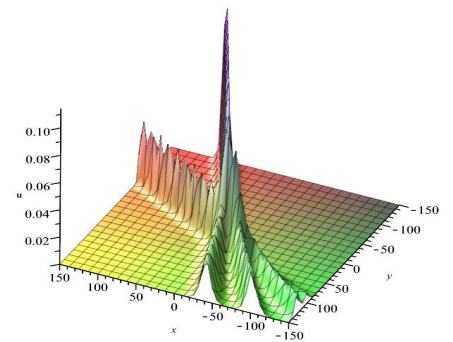

(a)

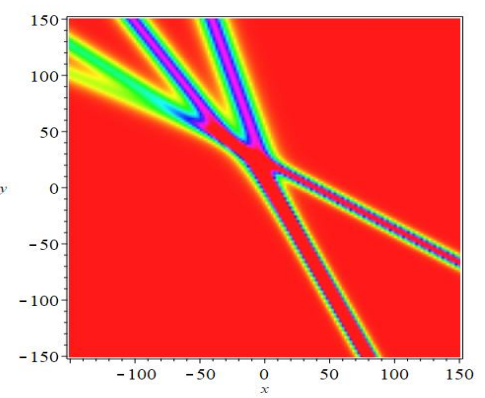

(d)

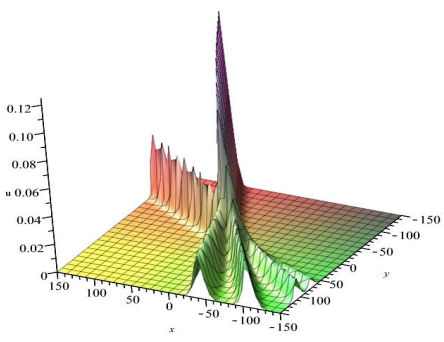

(b)

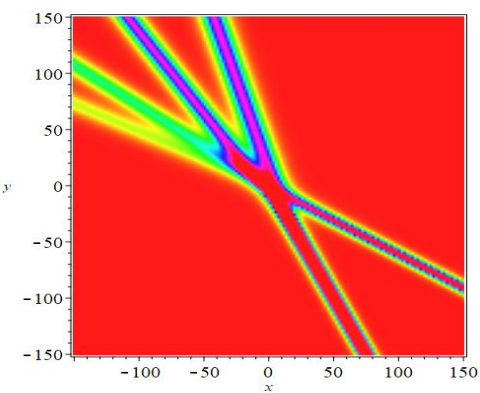

(e)

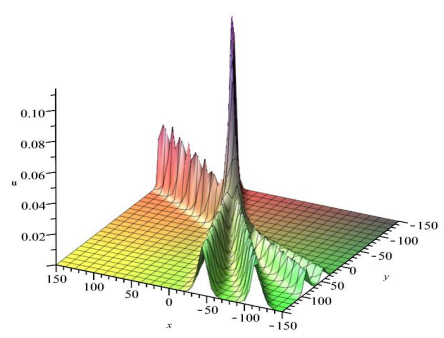

(c)

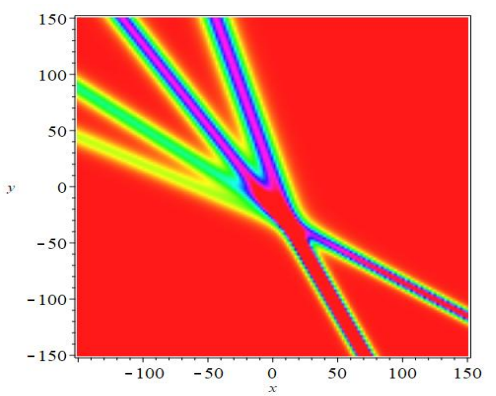

(f)

Figure 2: The interaction phenomena of two fission resonance Y-type solitons of (8) with $\alpha=1, \beta=1, k_{1}=\frac{3}{10}, k_{2}=\frac{1}{8}, k_{3}=\frac{1}{2}, k_{4}=$ $\frac{1}{4}, r_{1}=\frac{1}{2}, r_{2}=\frac{8021}{38400}+\frac{7 \sqrt{466}}{3200}, r_{3}=\frac{1}{4}, r_{4}=\frac{11}{64}, \phi_{1}=0, \phi_{2}=0, \phi_{3}=0, \phi_{4}=0$ when $t=-500$ in (a) and (d), $t=0$ in (b) and (e),
$t=500$ in (c) and (f), respectively. 


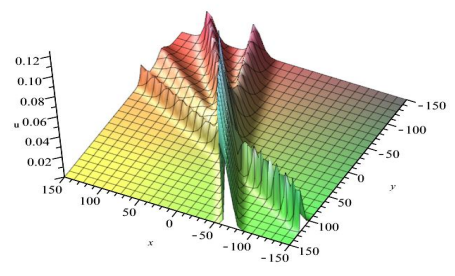

(a)

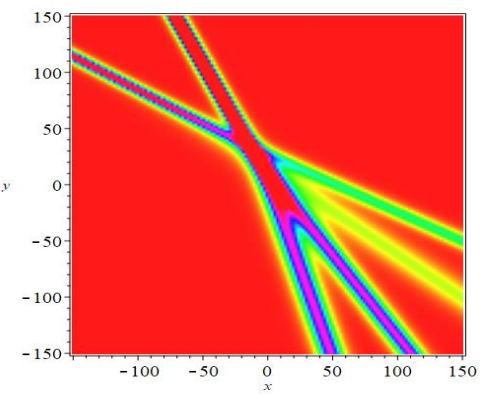

(d)

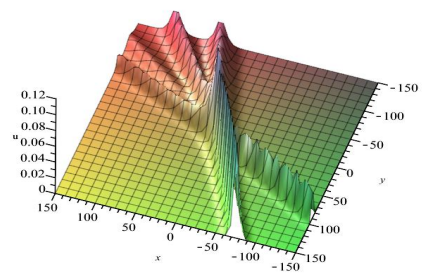

(b)

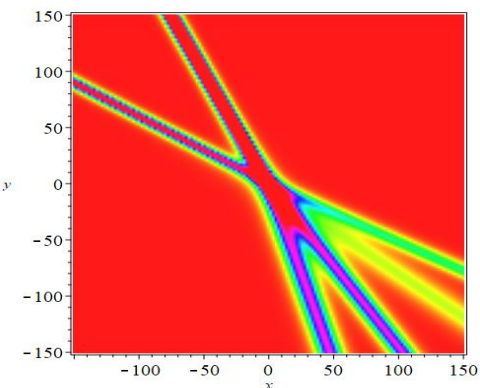

(e)

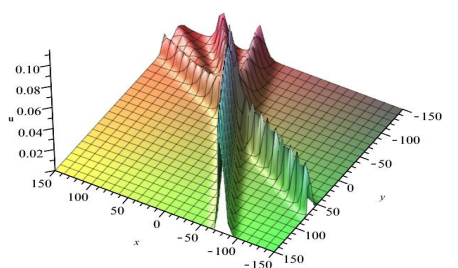

(c)

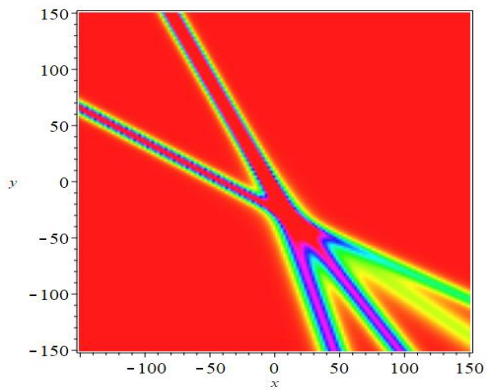

(f)

Figure 3: The interaction phenomena of two fusion resonance Y-type solitons of (8) with $\alpha=1, \beta=1, k_{1}=\frac{3}{10}, k_{2}=\frac{1}{8}, k_{3}=\frac{1}{2}, k_{4}=$ $\frac{1}{4}, r_{1}=\frac{1}{2}, r_{2}=\frac{8021}{38400}-\frac{7 \sqrt{466}}{3200}, r_{3}=\frac{1}{4}, r_{4}=\frac{5}{64}, \phi_{1}=0, \phi_{2}=0, \phi_{3}=0, \phi_{4}=0$ when $t=-500$ in (a) and (d), $t=0$ in (b) and (e),
$t=500$ in (c) and (f), respectively.

$$
\begin{aligned}
f_{3}=1 & +e^{\eta_{1}}+e^{\eta_{1}+\eta_{3}+A_{13}}+e^{\eta_{2}}+e^{\eta_{1}+\eta_{4}+A_{14}}+e^{\eta_{1}+\eta_{3}+A_{13}+\eta_{4}+A_{14}+A_{34}}+e^{\eta_{3}}+e^{\eta_{2}+\eta_{3}+A_{23}} \\
& +e^{\eta_{4}}+e^{\eta_{2}+\eta_{4}+A_{24}}+e^{\eta_{2}+\eta_{3}+A_{23}+\eta_{4}+A_{24}+A_{34}}+e^{\eta_{3}+\eta_{4}+A_{34}}
\end{aligned}
$$

where

$$
\begin{aligned}
r_{2}= & \frac{\beta k_{1}^{3}-3 \beta k_{1}^{2} k_{2}+2 \beta k_{1} k_{2}^{2}+2 \alpha r_{1}}{2 \alpha k_{1}} k_{2} \\
& \pm \frac{\sqrt{\left(k_{1}-k_{2}\right)^{2} k_{1}\left[\left(\beta^{2}-20 \alpha\right) k_{1}^{3}-4 k_{1} k_{2}\left(k_{1}-k_{2}\right)\left(\beta^{2}-5 \alpha\right)+12 \beta \alpha r_{1}\right]}}{2 \alpha k_{1}} k_{2}
\end{aligned}
$$

and

$$
\frac{k_{3}}{k_{4}}=\frac{r_{3}}{r_{4}}=\frac{w_{3}}{w_{4}} \neq \pm 1
$$

The interaction between 2-resonant Y-type soliton and a line soliton is shown in Fig. 6 .

Based on module resonant and constraints condition (7), a method to directly obtain solutions on resonant $\mathrm{Y}$-shape soliton and breather wave interactions is proposed. The following conditions are set on the N-soliton solution (3):

$$
\begin{aligned}
& e^{A_{j s}}=0, \eta_{L+2 p-1}=\eta_{L+2 p}^{*}, \\
& (1 \leq j<s \leq L, 1 \leq p \leq P, N=L+2 P) .
\end{aligned}
$$

If $L=2, P=1$, we will get a hybrid solution of a breather wave and 2-resonant Y-type soliton of equation (1), which is expressed as

$$
u_{4}=2\left(\ln f_{3}\right)_{x x},
$$




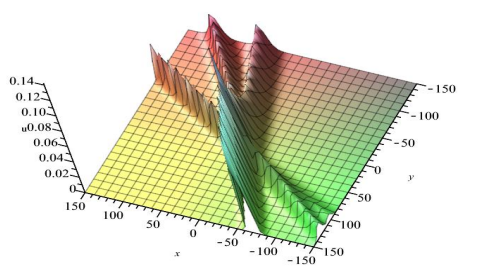

(a)

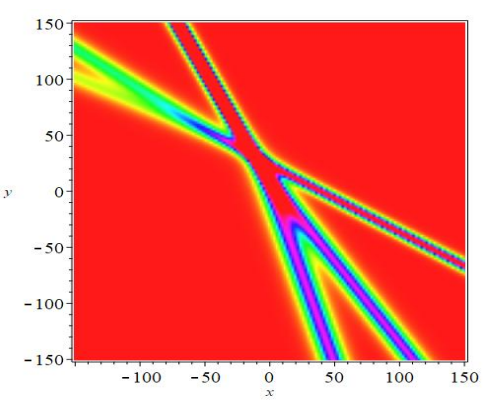

(d)

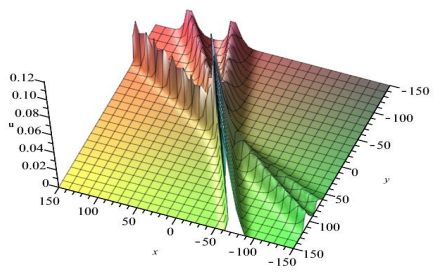

(b)

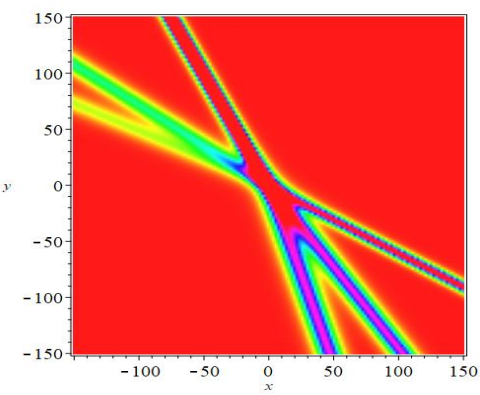

(e)

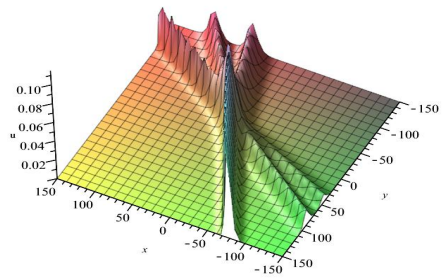

(c)

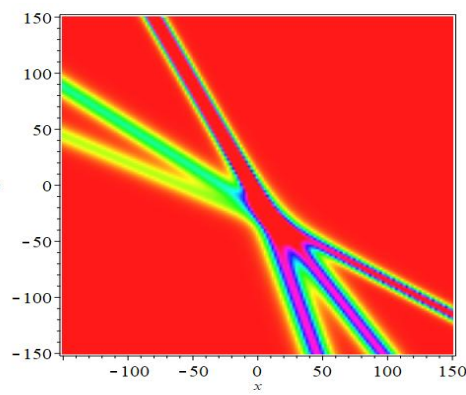

(f)

Figure 4: The interaction phenomena of one fission with one fusion resonance Y-type soliton of (8) with $\alpha=1, \beta=1, k_{1}=\frac{3}{10}, k_{2}=$ $\frac{1}{8}, k_{3}=\frac{1}{2}, k_{4}=\frac{1}{4}, r_{1}=\frac{1}{2}, r_{2}=\frac{8021}{38400}+\frac{7 \sqrt{466}}{3200}, r_{3}=\frac{1}{4}, r_{4}=\frac{5}{64}, \phi_{1}=0, \phi_{2}=0, \phi_{3}=0, \phi_{4}=0$ when $t=-500$ in (a) and (d), $t=0$ in
(b) and (e), $t=500$ in (c) and (f), respectively.

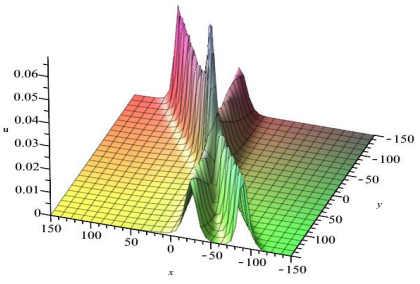

(a)

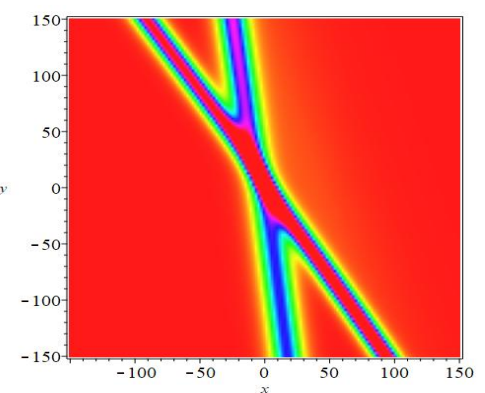

(d)

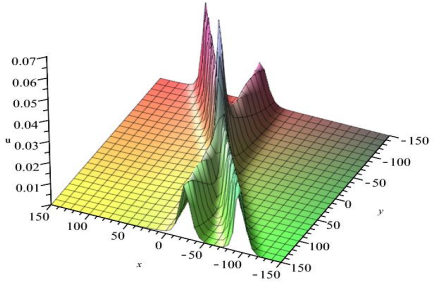

(b)

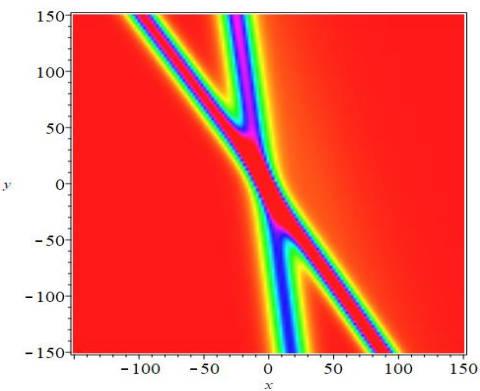

(e)

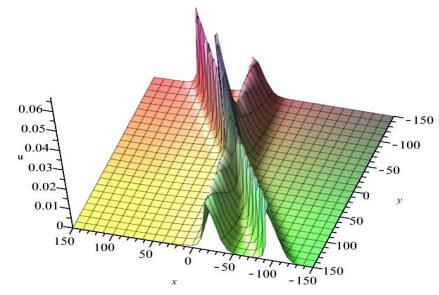

(c)

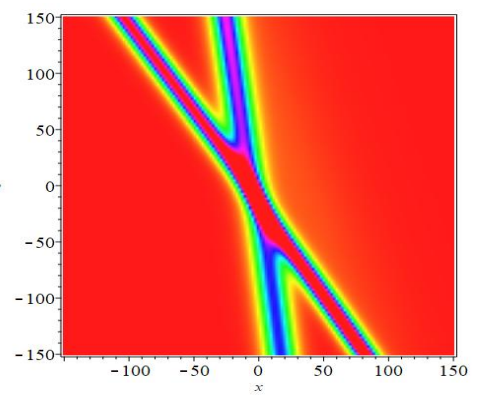

(f) Figure 5: Resonant X-type soliton of (9) with $\alpha=5, \beta=5, k_{1}=\frac{1}{2}, k_{2}=\frac{1}{4}, k_{3}=\frac{1}{5}, r_{1}=\frac{1}{5}, r_{2}=\frac{1}{10}+\frac{9 \sqrt{5}}{320}, r_{3}=\frac{83}{1000}-\frac{27 \sqrt{5}}{1000}, \phi_{1}=$
$0, \phi_{2}=0, \phi_{3}=0$ when $t=-100$ in (a) and (d), $t=0$ in (b) and (e), $t=100$ in (c) and (f), respectively. 
where

$$
k_{3}=k_{4}^{*}, r_{3}=r_{4}^{*}, \phi_{3}=\phi_{4}^{*}
$$

and $f_{3}$ and $r_{2}$ are constrained by (12) and (13).

It can be observed from Fig. 7 that the interaction between 2-resonant Y-type soliton and a breather wave is not affected by time, and the 2-resonant Y-type soliton moves only in the horizontal direction.

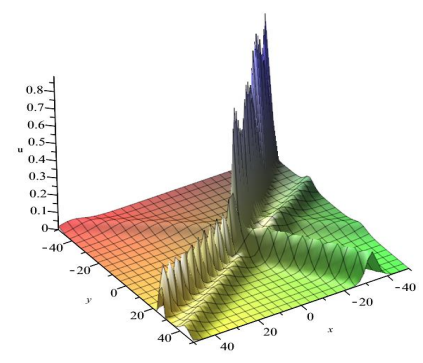

(a)

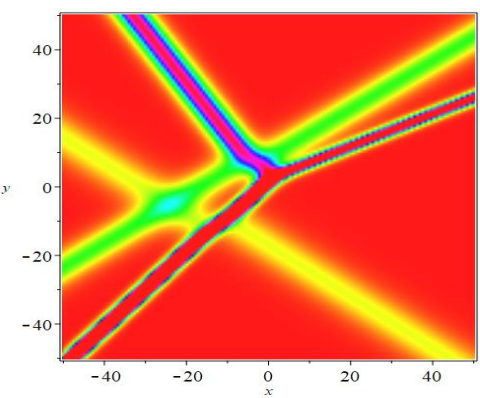

(d)

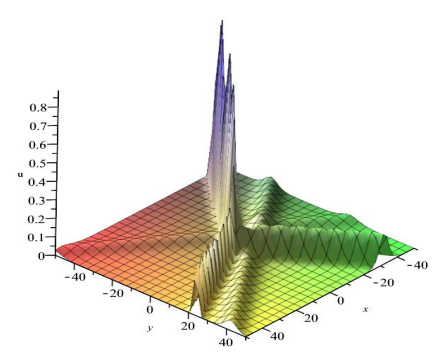

(b)

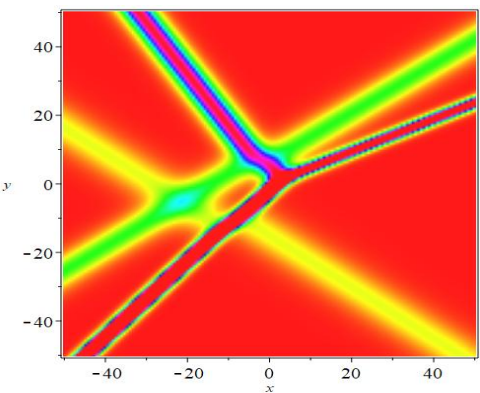

(e)

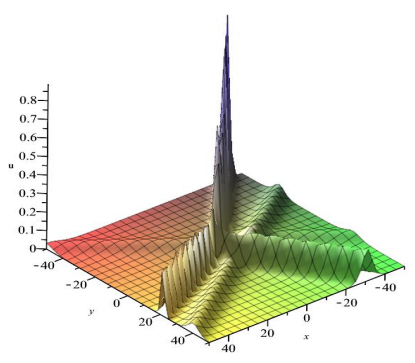

(c)

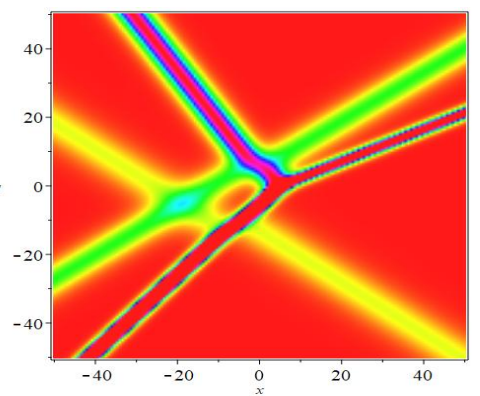

(f)

Figure 6: The interaction between a line soliton and 2-resonant Y-type soliton of (11) with $\alpha=-1, \beta=-1, k_{1}=-\frac{4}{3}, k_{2}=-\frac{3}{4}, k_{3}=$ $-\frac{1}{3}, k_{4}=\frac{1}{4}, r_{1}=\frac{6}{5}, r_{2}=\frac{683}{960}+\frac{7 \sqrt{14430}}{960}, r_{3}=\frac{1}{54}+\frac{\sqrt{38481}}{432}, r_{4}=\frac{1}{3}, \phi_{1}=0, \phi_{2}=0, \phi_{3}=-3, \phi_{4}=5$ when $t=-50$ in (a) and (d), $t=0$
in (b) and (e), $t=50$ in (c) and (f), respectively.

\section{Interaction between resonant Y-shape soliton solution and lump solution}

Based on constraint condition (7) and usual long-wave limit method, an approach to obtain interaction between high-order lump waves and resonant Y-type solitons is proposed directly. The following limiting conditions are set on the N-soliton solution(3):

$$
\begin{aligned}
& k_{2 m-1}=k_{2 m}^{*}=K_{2 m-1} \delta, r_{2 m-1}=r_{2 m}^{*}=R_{2 m-1} \delta, \phi_{2 m-1}=\phi_{2 m}=\pi i \\
& \delta \rightarrow 0, e^{A_{j s}}=0, N=2 L+P,(1 \leq m \leq L, 2 L<j<s \leq N) .
\end{aligned}
$$

Referring to an interesting work [38], we can know that the coordinates of lump's centre before and after interacting with resonant Y-shape soliton are given by parameters $\left\{K_{2 m-1}, R_{2 m-1}, K_{2 m}, R_{2 m}\right\}$. Next, we use $\left\{x_{b}, y_{b}\right\}$ to represent before the interaction, $\left\{x_{a}, y_{a}\right\}$ to represent after the interac- 


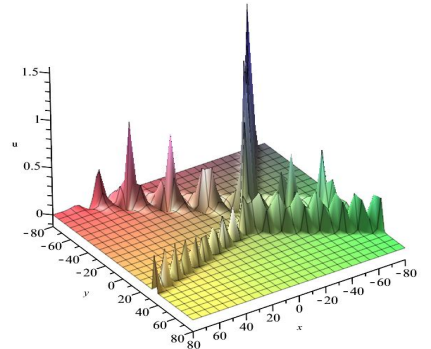

(a)

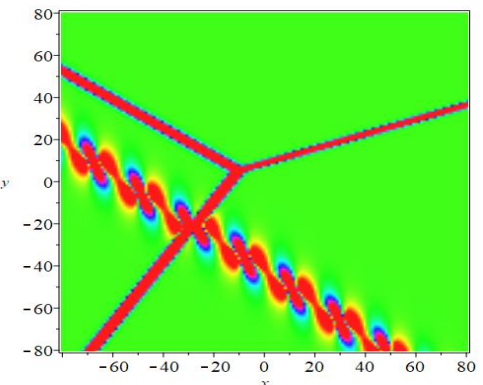

(d)

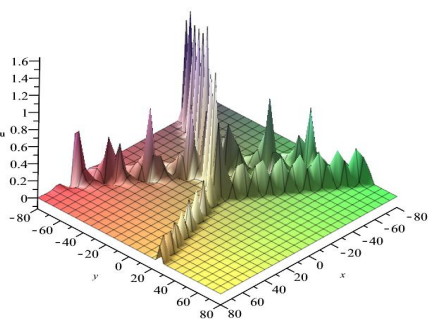

(b)

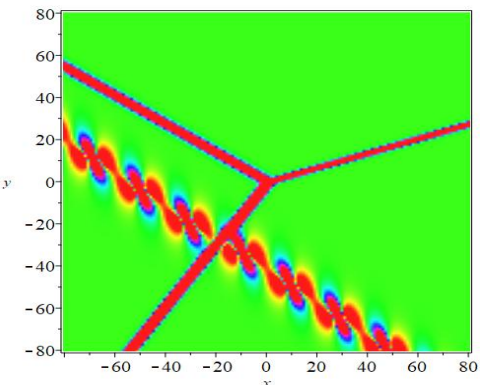

(e)

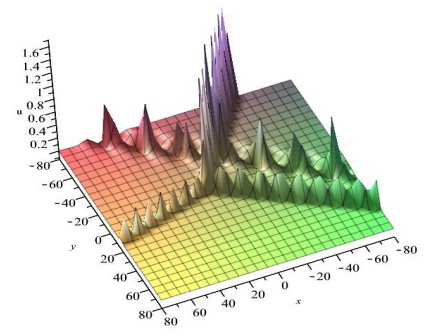

(c)

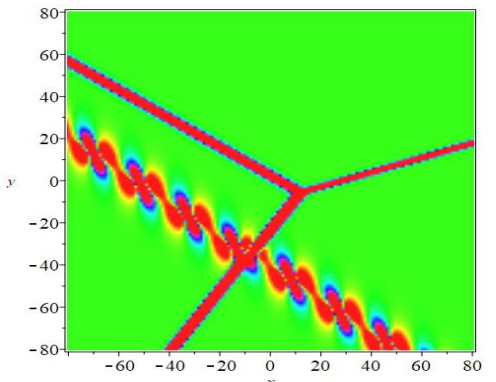

(f)

Figure 7: The interaction between a breather wave and 2-resonant Y-type soliton of (14) with $\alpha=-3, \beta=3, k_{1}=-\frac{16}{9}, k_{2}=-\frac{7}{8}, k_{3}=$ $\frac{1}{5}-\frac{i}{3}, k_{4}=\frac{1}{5}+\frac{i}{3}, r_{1}=\frac{6}{5}, r_{2}=\frac{124747}{207360}+\frac{91 \sqrt{108915}}{15552}, r_{3}=\frac{1}{4}, r_{4}=\frac{1}{4}, \phi_{1}=0, \phi_{2}=0, \phi_{3}=10, \phi_{4}=10$ when $t=-30$ in (a) and (d),
$t=0$ in (b) and (e), $t=30$ in (c) and (f), respectively. tion.

$$
\begin{aligned}
& x_{b}=\frac{\alpha R_{2 m-1} R_{2 m}}{36 K_{2 m-1} K_{2 m}} t+\sum_{s=2 L+1}^{N} h_{b}\left(\lambda_{s}\right) \Phi_{s} \\
& y_{b}=-\frac{\alpha\left(K_{2 m-1} R_{2 m}+K_{2 m} R_{2 m-1}\right)}{36 K_{2 m-1} K_{2 m}} t+\sum_{s=2 L+1}^{N} h_{b}\left(\lambda_{s}\right) \Theta_{s}, \\
& x_{a}=\frac{\alpha R_{2 m-1} R_{2 m}}{36 K_{2 m-1} K_{2 m}} t+\sum_{s=2 L+1}^{N} h_{a}\left(\lambda_{s}\right) \Phi_{s} \\
& y_{a}=-\frac{\alpha\left(K_{2 m-1} R_{2 m}+K_{2 m} R_{2 m-1}\right)}{36 K_{2 m-1} K_{2 m}} t+\sum_{s=2 L+1}^{N} h_{a}\left(\lambda_{s}\right) \Theta_{s},
\end{aligned}
$$

where

$$
\begin{gathered}
\lambda_{s}=\frac{\alpha k_{s} R_{2 m-1} R_{2 m}}{36 K_{2 m-1} K_{2 m}}-\frac{\alpha r_{s}\left(K_{2 m-1} R_{2 m}+K_{2 m} R_{2 m-1}\right)}{36 K_{2 m-1} K_{2 m}}+\frac{-k_{s}^{6}+\beta k_{s}^{3} r_{s}+\alpha r_{s}^{2}}{36 k_{s}}, \\
\Phi_{s}=\frac{-b_{2 m-1, s} R_{2 m}+b_{2 m, s} R_{2 m-1}}{K_{2 m-1} R_{2 m}-R_{2 m-1} K_{2 m}}, \Theta_{s}=\frac{b_{2 m-1, s} K_{2 m}-b_{2 m, s} K_{2 m-1}}{K_{2 m-1} R_{2 m}-R_{2 m-1} K_{2 m}}, \\
h_{b}(x)=\left\{\begin{array}{l}
1, x<0, \\
0, x \geq 0,
\end{array} h_{a}(x)=\left\{\begin{array}{l}
0, x \leq 0, \\
1, x>0,
\end{array}\right.\right.
\end{gathered}
$$


and

$$
b_{j s}= \begin{cases}\frac{6 \beta K_{j}^{2} K_{s}^{2}\left(K_{j} R_{s}+K_{s} R_{j}\right)}{\alpha\left(K_{j} R_{s}-K_{s} R_{j}\right)^{2}}, & 1 \leq j<s<2 L, \\ \frac{-6 k_{s}^{2} K_{j}^{2}\left[\left(-5 k_{s}^{3}+\beta r_{s}\right) K_{j}+\beta k_{s} R_{j}\right]}{\left(-5 k_{s}^{6}+2 \beta r_{s} k_{s}^{3}-\alpha r_{s}^{2}\right) K_{j}^{2}+k_{s} R_{j} K_{j}\left(\beta k_{s}^{3}+2 \alpha r_{s}\right)-\alpha k_{s}^{2} R_{j}^{2}}, & 1 \leq j \leq 2 L, s>2 L .\end{cases}
$$

What's more, the height of the lump solution will not increase or decrease before and after its interaction with one resonant Y-shape soliton. It is a fixed value, recorded as $h_{u}$.

$$
h_{u}=\frac{2 \alpha\left(K_{2 m-1} R_{2 m}-K_{2 m} R_{2 m-1}\right)^{2}}{3 \beta K_{2 m-1} K_{2 m}\left(K_{2 m-1} R_{2 m}+K_{2 m} R_{2 m-1}\right)} .
$$

If $L=1, P=2$, we will get a hybrid solution of one lump solution and one 2-resonant Y-type soliton. Under condition (15), the function $f$ in (3) becomes

$$
f_{4}=\theta_{1} \theta_{2}+b_{12}+\left(\theta_{1} \theta_{2}+b_{23} \theta_{1}+b_{13} \theta_{2}+b_{13} b_{23}+b_{12}\right) e^{\eta_{3}}+\left(\theta_{1} \theta_{2}+b_{24} \theta_{1}+b_{14} \theta_{2}+b_{14} b_{24}+b_{12}\right) e^{\eta_{4}},
$$

where $\theta_{j}=K_{j} x+R_{j} y+\frac{\alpha R_{j}^{2}}{36 K_{j}} t$.

Then, bring a function (16) into the logarithmic transformation (2), we can gain interaction between one lump and one 2-resonant Y-shape soliton of $u$.

Taking the parameters $\alpha=36, \beta=36, K_{1}=-\frac{6}{5}+\frac{6}{5} i, K_{2}=-\frac{6}{5}-\frac{6}{5} i, R_{1}=\frac{4}{3}, R_{2}=\frac{4}{3}, k_{3}=$ $\frac{5}{4}, r_{3}=\frac{1}{2}, k_{4}=\frac{3}{10}, r_{4}=\frac{1701}{8000}+\frac{19 \sqrt{16246}}{8000}, \phi_{1}=\pi i, \phi_{2}=\pi i, \phi_{3}=0, \phi_{4}=0$, Fig. 8 shows dynamic process of the interaction between a single lump solution and a resonance Y-type soliton. Before and after interaction, motion path of the single lump solution is $y=\frac{9}{5} x$ and $y=\frac{9}{5} x-\frac{74378116249560}{17368865139001}$, respectively.

From Fig. 8, one can see that after lump wave collides with the resonant Y-type soliton, the velocity, shape and amplitude do not change and continue to propagate, but the trajectory is offset. Next, we can study more complex resonant mixed solutions in the same way.

If $L=2, P=2$, we will get a hybrid solution of two lumps and one 2-resonant Y-type soliton. Under condition (15), the function $f$ in (3) becomes

$$
\begin{aligned}
& f_{5}=\theta_{1} \theta_{2} \theta_{3} \theta_{4}+b_{12} \theta_{3} \theta_{4}+b_{13} \theta_{2} \theta_{4}+b_{14} \theta_{2} \theta_{3}+b_{23} \theta_{1} \theta_{4}+b_{24} \theta_{1} \theta_{3}+b_{34} \theta_{1} \theta_{2}+b_{12} b_{34}+b_{13} b_{24}+ \\
& b_{14} b_{23}+\left(b_{15} b_{25} b_{35} b_{45}+b_{15} b_{25} b_{35} \theta_{4}+b_{15} b_{25} b_{45} \theta_{3}+b_{15} b_{25} \theta_{3} \theta_{4}+b_{15} b_{35} b_{45} \theta_{2}+b_{15} b_{35} \theta_{2} \theta_{4}\right. \\
& +b_{15} b_{45} \theta_{2} \theta_{3}+b_{15} \theta_{2} \theta_{3} \theta_{4}+b_{25} b_{35} b_{45} \theta_{1}+b_{25} b_{35} \theta_{1} \theta_{4}+b_{25} b_{45} \theta_{1} \theta_{3}+b_{25} \theta_{1} \theta_{3} \theta_{4}+b_{35} b_{45} \theta_{1} \theta_{2} \\
& +b_{35} \theta_{1} \theta_{2} \theta_{4}+b_{45} \theta_{1} \theta_{2} \theta_{3}+\theta_{1} \theta_{2} \theta_{3} \theta_{4}+b_{12} b_{35} b_{45}+b_{12} b_{35} \theta_{4}+b_{12} b_{45} \theta_{3}+b_{12} \theta_{3} \theta_{4}+b_{13} b_{25} b_{45} \\
& +b_{13} b_{25} \theta_{4}+b_{13} b_{45} \theta_{2}+b_{13} \theta_{2} \theta_{4}+b_{14} b_{25} b_{35}+b_{14} b_{25} \theta_{3}+b_{14} b_{35} \theta_{2}+b_{14} \theta_{2} \theta_{3}+b_{15} b_{23} b_{45} \\
& +b_{15} b_{23} \theta_{4}+b_{15} b_{24} b_{35}+b_{15} b_{24} \theta_{3}+b_{15} b_{25} b_{34}+b_{15} b_{34} \theta_{2}+b_{23} b_{45} \theta_{1}+b_{23} \theta_{1} \theta_{4}+b_{24} b_{35} \theta_{1} \\
& \left.+b_{24} \theta_{1} \theta_{3}+b_{25} b_{34} \theta_{1}+b_{34} \theta_{1} \theta_{2}+b_{12} b_{34}+b_{13} b_{24}+b_{14} b_{23}\right) e^{\eta_{5}} \\
& +\left(b_{16} b_{26} b_{36} b_{46}+b_{16} b_{26} b_{36} \theta_{4}+b_{16} b_{26} b_{46} \theta_{3}+b_{16} b_{26} \theta_{3} \theta_{4}+b_{16} b_{36} b_{46} \theta_{2}+b_{16} b_{36} \theta_{2} \theta_{4}+\right. \\
& b_{16} b_{46} \theta_{2} \theta_{3}+b_{16} \theta_{2} \theta_{3} \theta_{4}+b_{26} b_{36} b_{46} \theta_{1}+b_{26} b_{36} \theta_{1} \theta_{4}+b_{26} b_{46} \theta_{1} \theta_{3}+b_{26} \theta_{1} \theta_{3} \theta_{4}+b_{36} b_{46} \theta_{1} \theta_{2} \\
& +b_{36} \theta_{1} \theta_{2} \theta_{4}+b_{46} \theta_{1} \theta_{2} \theta_{3}+\theta_{1} \theta_{2} \theta_{3} \theta_{4}+b_{12} b_{36} b_{46}+b_{12} b_{36} \theta_{4}+b_{12} b_{46} \theta_{3}+b_{12} \theta_{3} \theta_{4}+ \\
& b_{13} b_{26} b_{46}+b_{13} b_{26} \theta_{4}+b_{13} b_{46} \theta_{2}+b_{13} \theta_{2} \theta_{4}+b_{14} b_{26} b_{36}+b_{14} b_{26} \theta_{3}+b_{14} b_{36} \theta_{2}+b_{14} \theta_{2} \theta_{3}+ \\
& b_{16} b_{23} b_{46}+b_{16} b_{23} \theta_{4}+b_{16} b_{24} b_{36}+b_{16} b_{24} \theta_{3}+b_{16} b_{26} b_{34}+b_{16} b_{34} \theta_{2}+b_{23} b_{46} \theta_{1}+b_{23} \theta_{1} \theta_{4}+ \\
& \left.b_{24} b_{36} \theta_{1}+b_{24} \theta_{1} \theta_{3}+b_{26} b_{34} \theta_{1}+b_{34} \theta_{1} \theta_{2}+b_{12} b_{34}+b_{13} b_{24}+b_{14} b_{23}\right) e^{\eta_{6}},
\end{aligned}
$$


where $\theta_{j}=K_{j} x+R_{j} y+\frac{\alpha R_{j}^{2}}{36 K_{j}} t$.

Then, bring a function (17) into the logarithmic transformation (2), we can gain interaction between two lumps and one 2-resonant Y-shape soliton of $u$.

Taking parameters $\alpha=5, \beta=5, K_{1}=\frac{1}{2}+i, K_{2}=\frac{1}{2}-i, K_{3}=\frac{1}{4}+\frac{3}{5} i, K_{4}=\frac{1}{4}-$

$\frac{3}{5} i, R_{1}=-\frac{11}{5}, R_{2}=-\frac{11}{5}, R_{3}=-\frac{11}{10}, R_{4}=-\frac{11}{10}, k_{5}=\frac{11}{10}, k_{6}=-\frac{11}{10}, r_{5}=\frac{1}{2}, r_{6}=-\frac{4493}{1000}-$ $\frac{33 \sqrt{2453}}{1000}, \phi_{1}=\pi i, \phi_{2}=\pi i, \phi_{3}=\pi i, \phi_{4}=\pi i, \phi_{5}=0, \phi_{6}=0$, Fig. 9 shows the interaction between the 2-order lump solution and a resonance Y-type soliton.

\section{Conclusions}

In this paper, subject to N-soliton solutions of (2+1)-dimensional gCDGKS equation, we successfully construct resonant Y-shape soliton and their interactions with a number of other solutions. Firstly, by proposing a noval restrictive condition for the N-soliton solution, resonant $\mathrm{Y}$-shape soliton solution of (2) is discussed, especially the three interactions of two 2-resonant Y-type soliton solutions: two fusion, two fission and one fusion with one fission. By selecting appropriate parameters, a new double opening resonant Y-type soliton can be obtained. As illustrated in Fig. 5, it can be referred to as a resonant X-shape soliton because its shape resembles the letter "X". Secondly, combined with velocity resonance method and mode resonance method, interaction solutions of these resonant Y-shape solitons with line waves and breather solutions are gained. Finally, with the support of long wave limit method, the hybrid solution composed of resonant Y-shape soliton and higher order lump solutions is given, and the coordinates of lump's centre before and after interacting with resonant Y-shape soliton are derived.

\section{Acknowledgements}

The work is supported by the National Natural Science Foundation of China (project No. 11371086, 11671258, 11975145), the Fund of Science and Technology Commission of Shanghai Municipality (project No. 13ZR1400100), the Fund of Donghua University, institute for nonlinear sciences and the Fundamental Research Funds for the Central Universities with contract number $2232021 \mathrm{G}-13$.

\section{Compliance with ethical standards Conflict}

\section{Conflict of interest}

There are no conflicts of interest to this work.

\section{Data Availability Statements}

Data sharing not applicable to this article as no datasets were generated. 


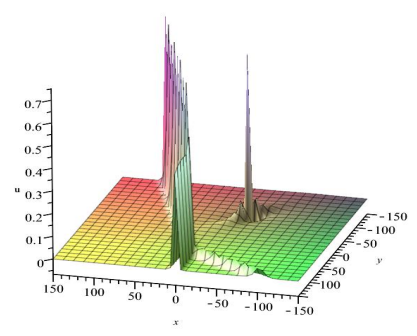

(a)

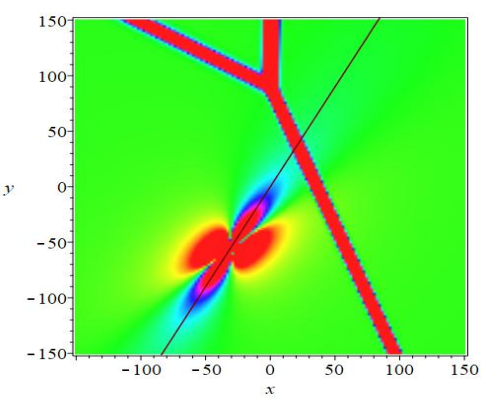

(d)

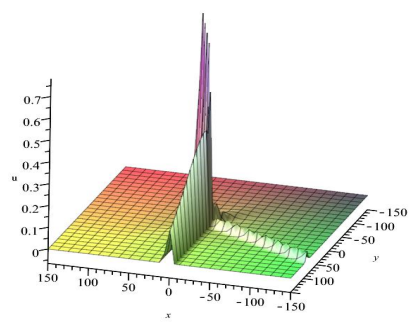

(g)

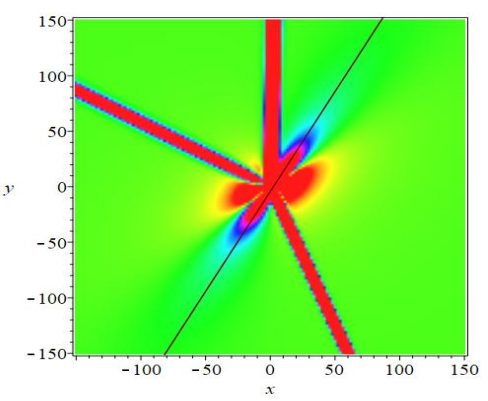

(j)

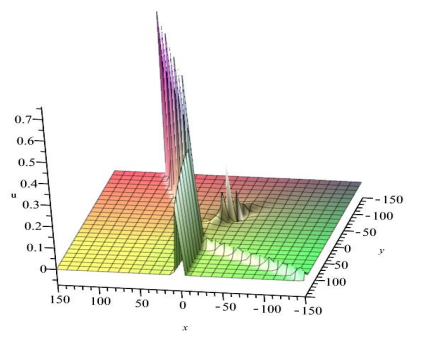

(b)

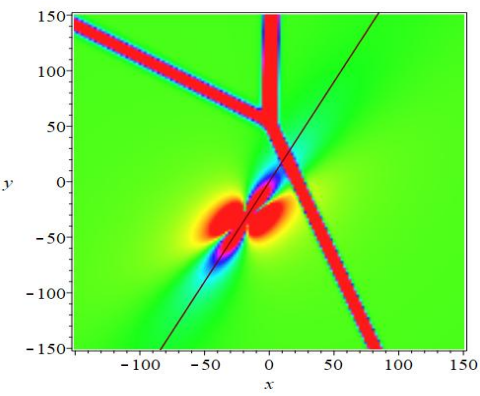

(e)

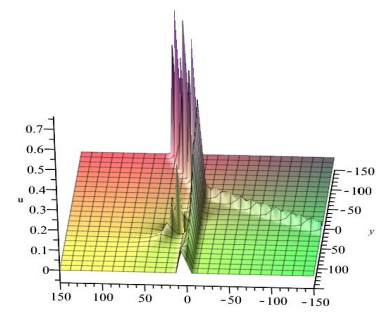

(h)

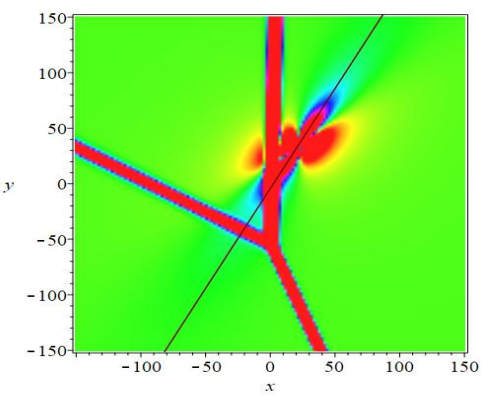

(k)

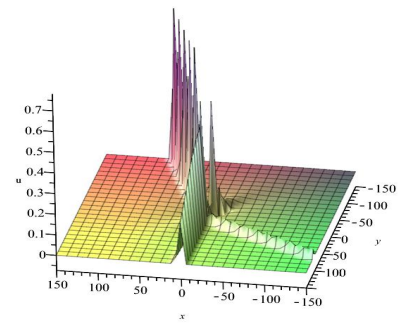

(c)

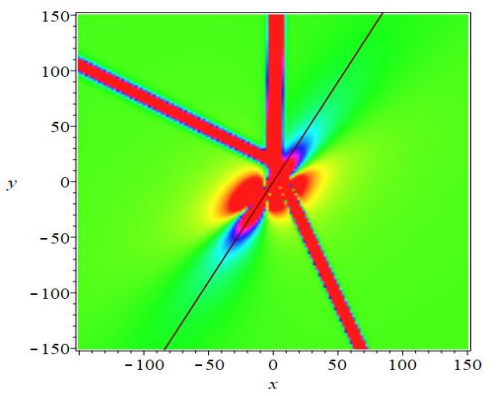

(f)

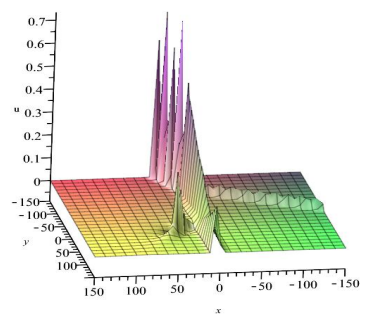

(i)

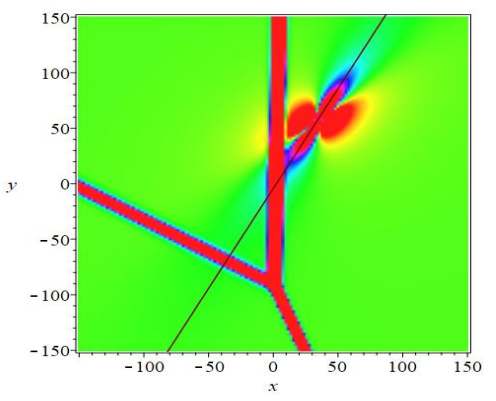

(1)

Figure 8: Three-dimensional plots and density plots of interaction between a single lump solution and a resonance Y-type soliton with $\alpha=36, \beta=36, K_{1}=-\frac{6}{5}+\frac{6}{5} i, K_{2}=-\frac{6}{5}-\frac{6}{5} i, R_{1}=\frac{4}{3}, R_{2}=\frac{4}{3}, k_{3}=\frac{5}{4}, r_{3}=\frac{1}{2}, k_{4}=\frac{3}{10}, r_{4}=\frac{1701}{8000}+\frac{19 \sqrt{16246}}{8000}, \phi_{1}=\pi i, \phi_{2}=\pi i, \phi_{3}=$ $0, \phi_{4}=0$ when $t=-50$ in (a) and (g), $t=-30$ in (b) and (h), $t=-10$ in (c) and (i), $t=0$ in (d) and (j), $t=30$ in (e) and (k), $t=50$ in (e) and (1), respectively. 


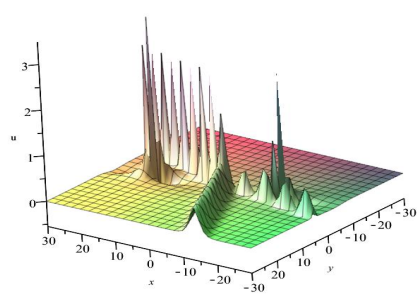

(a)

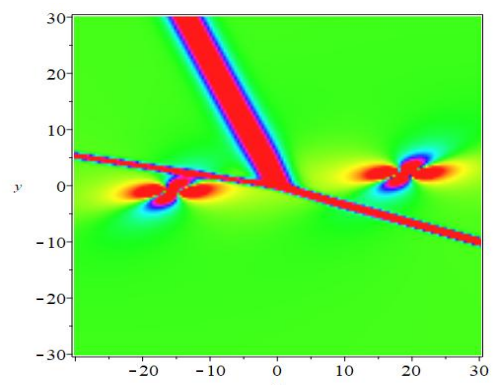

(b)

Figure 9: Three-dimensional plots and density plots of the interaction between two lumps and one resonance Y-type soliton with $\alpha=5, \beta=5, K_{1}=\frac{1}{2}+i, K_{2}=\frac{1}{2}-i, K_{3}=\frac{1}{4}+\frac{3}{5} i, K_{4}=\frac{1}{4}-\frac{3}{5} i, R_{1}=-\frac{11}{5}, R_{2}=-\frac{11}{5}, R_{3}=-\frac{11}{10}, R_{4}=-\frac{11}{10}, k_{5}=\frac{11}{10}, k_{6}=-\frac{11}{10}, r_{5}=$ $\frac{1}{2}, r_{6}=-\frac{4493}{1000}-\frac{33 \sqrt{2453}}{1000}, \phi_{1}=\pi i, \phi_{2}=\pi i, \phi_{3}=\pi i, \phi_{4}=\pi i, \phi_{5}=0, \phi_{6}=0$ when $t=0$.

\section{References}

[1] Michel Remoissenet. Solitons in nonlinear transmission lines. In Waves Called Solitons, pages 37-64. Springer, 1996.

[2] Sen-Yue Lou. Soliton molecules and asymmetric solitons in three fifth order systems via velocity resonance. Journal of Physics Communications, 4(4):041002, 2020.

[3] Xue-Ping Cheng, Sen-Yue Lou, Yun-Qing Yang, Peng Li, and Sheng-Wen Qi. The nsoliton molecule for the combined $(2 \mathrm{n}+1)$ th-order lax's kdv equation. Results in Physics, 18:103184, 2020.

[4] Hong-Cai Ma, Huai-Yu Huang, and Ai-Ping Deng. Solitons and soliton molecules in two nonlocal alice-bob fifth-order $\mathrm{kdv}$ systems. International Journal of Theoretical Physics, 60(8):3051-3062, 2021.

[5] Hong-Cai Ma, Yu-Xin Wang, and Ai-Ping Deng. Soliton molecules and asymmetric solitons of the extended lax equation via velocity resonance. Chinese Physics B, 2021.

[6] Hong-Cai Ma, Shu-Pan Yue, and Ai-Ping Deng. D'alembert wave, the hirota conditions and soliton molecule of a new generalized kdv equation. Journal of Geometry and Physics, 172:104413, 2022.

[7] KW Chow, RHJ Grimshaw, and E Ding. Interactions of breathers and solitons in the extended korteweg-de vries equation. Wave Motion, 43(2):158-166, 2005.

[8] Wei Liu, Abdul-Majid Wazwaz, and Xiao-Xiao Zheng. Families of semi-rational solutions to the kadomtsev-petviashvili i equation. Communications in Nonlinear Science and Numerical Simulation, 67:480-491, 2019.

[9] J Satsuma and MJ Ablowitz. Two-dimensional lumps in nonlinear dispersive systems. Journal of Mathematical Physics, 20(7):1496-1503, 1979.

[10] Yan Zhang, Yin-Ping Liu, and Xiao-Yan Tang. M-lump solutions to a (3+1)-dimensional nonlinear evolution equation. Computers \& Mathematics with Applications, 76(3):592-601, 2018. 
[11] Zhao-Wen Yan and Sen-Yue Lou. Special types of solitons and breather molecules for a $(2+1)$-dimensional fifth-order $\mathrm{kdv}$ equation. Communications in Nonlinear Science and Numerical Simulation, 91:105425, 2020.

[12] Zhao Zhang, Shu-Xin Yang, and Biao Li. Soliton molecules, asymmetric solitons and hybrid solutions for $(2+1)$-dimensional fifth-order $\mathrm{kdv}$ equation. Chinese Physics Letters, 36(12):120501, 2019.

[13] Hong-Cai Ma, Huai-Yu Huang, and Ai-Ping Deng. Soliton molecules and some interaction solutions for the (3+1)-dimensional jimbo-miwa equation. Journal of Geometry and Physics, 170:104362, 2021.

[14] Hong-Cai Ma, Huai-Yu Huang, and Ai-Ping Deng. Soliton molecules and some novel hybrid solutions for $(3+1)$-dimensional b-type kadomtsev-petviashvili equation. Modern Physics Letters B, 35(23):2150388, 2021.

[15] Hong-Cai Ma, Yu-Xin Wang, and Ai-Ping Deng. Soliton molecules and some novel mixed solutions for the extended caudrey-dodd-gibbon equation. Journal of Geometry and Physics, 168:104309, 2021.

[16] Zhong-Long Zhao and Ling-Chao He. M-lump, high-order breather solutions and interaction dynamics of a generalized (2+1)-dimensional nonlinear wave equation. Nonlinear Dynamics, 100(3):2753-2765, 2020.

[17] K Sakkaravarthi, T Kanna, M Vijayajayanthi, and M Lakshmanan. Multicomponent longwave-short-wave resonance interaction system: bright solitons, energy-sharing collisions, and resonant solitons. Physical Review E, 90(5):052912, 2014.

[18] Wei Liu, Xiao-Xiao Zheng, Chu Wang, and Sheng-Qi Li. Fission and fusion collision of highorder lumps and solitons in a (3+1)-dimensional nonlinear evolution equation. Nonlinear Dynamics, 96(4):2463-2473, 2019.

[19] Hui-Min Yin, Bo Tian, and Xin-Chao Zhao. Chaotic breathers and breather fission/fusion for a vector nonlinear schrödinger equation in a birefringent optical fiber or wavelength division multiplexed system. Applied Mathematics and Computation, 368:124768, 2020.

[20] Ai-Hua Chen. Multi-kink solutions and soliton fission and fusion of sharma-tasso-olver equation. Physics Letters A, 374(23):2340-2345, 2010.

[21] Yu-Feng Wang, Bo Tian, and Yan Jiang. Soliton fusion and fission in a generalized variablecoefficient fifth-order korteweg-de vries equation in fluids. Applied Mathematics and Computation, 292:448-456, 2017.

[22] Ai-Hua Chen and Fan-Fan Wang. Fissionable wave solutions, lump solutions and interactional solutions for the (2+1)-dimensional sawada-kotera equation. Physica Scripta, 94(5):055206, 2019.

[23] Chun-Ku Kuo and Wen-Xiu Ma. A study on resonant multi-soliton solutions to the (2+1)dimensional hirota-satsuma-ito equations via the linear superposition principle. Nonlinear Analysis, 190:111592, 2020. 
[24] Jia-Heng Li, Qing-Qing Chen, and Biao Li. Resonance y-type soliton solutions and some new types of hybrid solutions in the $(2+1)$-dimensional sawada-kotera equation. Communications in Theoretical Physics, 73(4):045006, 2021.

[25] Miao-Miao Wang, Ze-Qun Qi, Jun-Chao Chen, and Biao Li. Resonance y-shaped soliton and interaction solutions in the (2+1)-dimensional b-type kadomtsev-petviashvili equation. International Journal of Modern Physics B, 35(21):2150222, 2021.

[26] Shu-Xin Yang, Zhao Zhang, and Biao Li. Y-shaped soliton solutions for the (2+1)-dimensional bidirectional sawada-kotera equation. Modern Physics Letters B, 35(33):2150488, 2021.

[27] Zhao Zhang, Ze-Qun Qi, and Biao Li. Fusion and fission phenomena for (2+ 1)-dimensional fifth-order kdv system. Applied Mathematics Letters, 116:107004, 2021.

[28] Wei-Qi Peng, Shou-Fu Tian, Li Zou, and Tian-Tian Zhang. Characteristics of the solitary waves and lump waves with interaction phenomena in a $(2+1)$-dimensional generalized caudrey-dodd-gibbon-kotera-sawada equation. Nonlinear Dynamics, 93(4):1841-1851, 2018.

[29] Gao-Fu Deng, Yi-Tian Gao, Jing-Jing Su, Cui-Cui Ding, and Ting-Ting Jia. Solitons and periodic waves for the $(2+1)$-dimensional generalized caudrey-dodd-gibbon-kotera-sawada equation in fluid mechanics. Nonlinear Dynamics, 99(2):1039-1052, 2020.

[30] BG Konopelchenko and VG Dubrovsky. Some new integrable nonlinear evolution equations in 2+1 dimensions. Physics Letters A, 102(1-2):15-17, 1984.

[31] Ce-Wen Cao, Yong-Tang Wu, and Xian-Guo Geng. On quasi-periodic solutions of the $2+1$ dimensional caudrey-dodd-gibbon-kotera-sawada equation. Physics Letters A, 256(1):5965, 1999.

[32] Jian-Hong Zhuang, Ya-Qing Liu, Xin Chen, Juan-Juan Wu, and Xiao-Yong Wen. Diverse solitons and interaction solutions for the (2+1)-dimensional cdgks equation. Modern Physics Letters B, 33(16):1950174, 2019.

[33] Run-Fa Zhang, Ming-Chu Li, Mohammed Albishari, Fu-Chang Zheng, and Zhong-Zhou Lan. Generalized lump solutions, classical lump solutions and rogue waves of the $(2+1)$ dimensional caudrey-dodd-gibbon-kotera-sawada-like equation. Applied Mathematics and Computation, 403:126201, 2021.

[34] Chang-Fu Liu and Zheng-De Dai. Exact soliton solutions for the fifth-order sawada-kotera equation. Applied Mathematics and Computation, 206(1):272-275, 2008.

[35] Hasibun Naher, Farah Aini Abdullah, and Syed Tauseef Mohyud-Din. Extended generalized riccati equation mapping method for the fifth-order sawada-kotera equation. AIP Advances, 3(5):052104, 2013.

[36] Yan-Feng Guo, Dong-Long Li, and Jun-Xia Wang. The new exact solutions of the fifth-order sawada-kotera equation using three wave method. Applied Mathematics Letters, 94:232-237, 2019 .

[37] Ryogo Hirota and Junkichi Satsuma. Soliton solutions of a coupled korteweg-de vries equation. Physics Letters A, 85(8-9):407-408, 1981. 
[38] Zhao Zhang, Xiang-Yu Yang, Wen-Tao Li, and Biao Li. Trajectory equation of a lump before and after collision with line, lump, and breather waves for $(2+1)$-dimensional kadomtsevpetviashvili equation. Chinese Physics B, 28(11):110201, 2019. 